\title{
PYRROC: the first functionalized cycloalkyne that facilitates isomer-free generation of organic molecules by SPAAC $\uparrow$
}

Cite this: Org. Biomol. Chem., 2015, 13, 3866

Received 2nd February 2015,

Accepted 16th February 2015

DOI: 10.1039/c5ob00212e

\author{
Corinna Gröst and Thorsten Berg*
}

www.rsc.org/obc

\begin{abstract}
We present the concept, synthesis, and kinetic characterization of PYRROC as the first functionalized cycloalkyne which cannot form isomers in the reaction with azides. In aqueous buffer, PYRROC displays unprecedented rate accelerations in SPAAC of three to four orders of magnitude, leading to rate constants exceeding $400 \mathrm{M}^{-1} \mathrm{~s}^{-1}$.
\end{abstract}

The Huisgen 1,3-dipolar cycloaddition between azides and alkynes $^{1}$ has gained tremendous attention in recent years. Catalysis by $\mathrm{Cu}(\mathrm{I})$ not only vastly accelerates the reaction, but also introduces a very high degree of regioselectivity for 1,4-triazoles over 1,5-triazoles. ${ }^{2,3}$ These favorable properties have made copper-catalyzed azide-alkyne cycloadditions (CuAAC) a centrally important reaction for the generation of bioactive molecules. ${ }^{4}$ However, copper ions are toxic to living cells, limiting the use of CuAAC in a cellular context. This limitation was overcome by strain-promoted azide-alkyne cycloaddition reactions (SPAAC), ${ }^{5,6}$ which generate triazoles in the absence of copper, allowing widespread applications related to the labeling of biomolecules. The reactivity of the first functionalized cyclooctyne $\mathrm{OCT}^{7}$ can be increased by placing fluorine in the propargylic position, or by introducing additional ring strain via $\mathrm{sp}^{2}$-hybridized centers or a fused cyclopropyl ring, as illustrated by difluorocyclooctyne (DIFO), ${ }^{8}$ dibenzocyclooctyne (DIBO),${ }^{9}$ and bicyclononyne (BCN), ${ }^{10}$ respectively (Fig. 1a).

A major shortcoming of SPAAC is that the degree of regioselectivity in the transition state of the reaction is usually low, resulting in the formation of isomeric products. For most functionalized cyclooctynes attached to a chemical entity $\mathrm{R}^{1}$ via their respective functional groups, this yields regioisomers in similar or equal ratios (Fig. 1b). Sterically hindered DIBO derivatives offer the opportunity to tailor regioselectivity by adding sterical hindrance on one of the phenyl rings. ${ }^{11}$ For

Institute or Organic Chemistry, University of Leipzig, Johannisallee 29, 04103 Leipzig, Germany. E-mail: tberg@uni-leipzig.de; Fax: (+49) 3419736599 $\dagger$ Electronic supplementary information (ESI) available: Synthesis and characterization of compounds, kinetic experiments, and methods. See DOI: 10.1039/ c5ob00212e a)<smiles>O=C(O)c1ccc(COC2C=CCCCCC2)cc1</smiles><smiles>O=C(O)COC1CCC=CC(F)(F)CC1</smiles><smiles>OC1Cc2ccccc2C=Cc2ccccc21</smiles>

DIBO
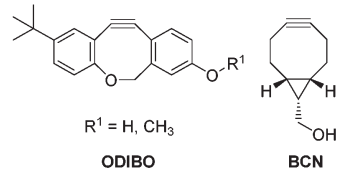

b)<smiles>[R20]Oc1ccc(COC2C=CCCCC2)cc1</smiles>

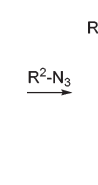

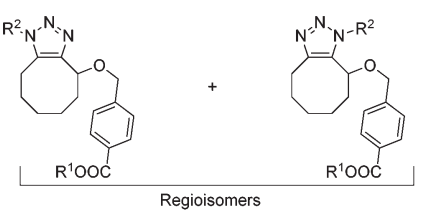

c)
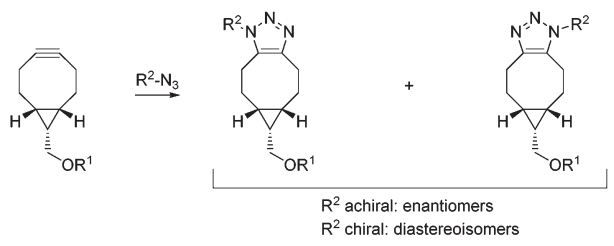

Fig. 1 SPAAC with known functionalized cycloalkynes usually leads to the formation of isomeric products. (a) Structures of some functionalized cycloalkynes. (b) SPAAC with OCT derivatized via its carboxylic acid group leads to the formation of regioisomers. (c) SPAAC with BCN derivatized via its hydroxy group leads to the formation of enantiomers or diastereomers, depending on the absence or presence of chiral centers in the azide.

ODIBO (Fig. 1a), the formation of only a single isomer was reported from the reaction with azides. ${ }^{12}$ However, given the potential for regioisomer formation by DIBO derivatives, high regioselectivity cannot generally be guaranteed for functionalized DIBO derivatives in the reaction with any given azide. The bicyclononyne $\mathrm{BCN}^{10}$ does not form regioisomers, but either enantiomers or diastereoisomers, depending on the presence of chirality in the azide (Fig. 1c).

Whilst the formation of isomers is typically not regarded as a drawback of the cycloalkynes currently used for labeling of biomolecules by SPAAC, ${ }^{13}$ it is a prohibitive feature for a new potential application of SPAAC: isomer-free, bioorthogonal synthesis of organic molecules in the cellular environment. An isomer-free approach would facilitate the intracellular 

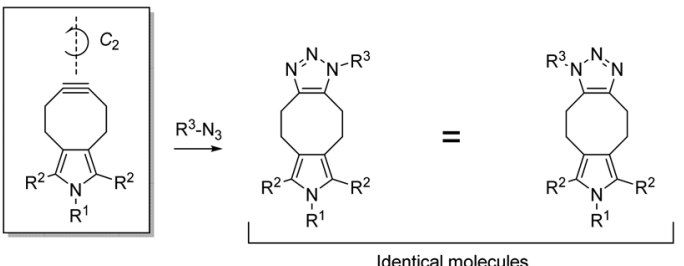

Fig. 2 Cycloalkynes (here: pyrrolocyclooctynes) which contain a $C_{2^{-}}$symmetry axis, either in a preferred conformation or in a conformational transition state, generate chemically uniform SPAAC products.

generation of large bioactive molecules with poor cell-permeability, from two smaller, more cell-permeable building blocks. Such large bioactive molecules could potentially serve as protein dimerizers, ${ }^{14}$ bivalent ligands addressing two domains within the same protein, or inhibitors of large protein-protein interfaces. ${ }^{15}$

To circumvent isomer formation in SPAAC, we propose the concept of symmetrically substituted, functionalized cycloalkynes (Fig. 2). Functionalized cycloalkynes that harbor a $C_{2}$-symmetry axis, either in a preferred conformation or in a predicted conformational transition state, form uniform reaction products with azides $\mathrm{R}^{3}-\mathrm{N}_{3}$ regardless of the relative orientation between cycloalkyne and azide during the reaction. Key to preventing the possibility of generating isomers in SPAAC is the attachment of the substituent $\mathrm{R}^{1}$ at a nitrogen atom, which is symmetrically placed in an odd-membered ring fused at the 5,6-position of the cyclooctyne. Any substituents must be placed consistent with the outlined concept of $C_{2}$-symmetry. In this communication, we report on the synthesis and kinetic characterization of the first functionalized cyclooctyne that meets these criteria. We demonstrate that the new cycloalkyne, a pyrrolocyclooctyne, is well suited for the isomer-free generation of large organic molecules in the aqueous environment, providing good yields within a short time frame.

The synthesis of the pyrrolocyclooctyne started with the nitration of 1,4-cyclooctadiene with silver nitrite and TEMPO to obtain compound $\mathbf{1}$, which is subjected to the Barton-Zard reaction, leading to the formation of pyrrolocyclooctene 2 (Scheme 1). ${ }^{16,17}$ Ester hydrolysis, decarboxylation, and $\mathrm{N}$-Boc protection afforded 3 in very good yield. To reduce the reactivity of 3 , which is prone to decomposition, it was converted into the diester 4. In line with the literature, ${ }^{18}$ it was essential to add the solution of the 2,5-dilithiated pyrrole based on 3 to a solution of electrophile to obtain the 2,5-disubstituted pyrrole 4. ${ }^{19}$ Attempts to convert 2 into 4 directly were not successful. Bromination of the alkene moiety of $\mathbf{4}$ and removal of the protecting group led to dibromide $\mathbf{5}$, which was reacted with tertbutyl(3-iodopropoxy)dimethylsilane $\mathbf{6}$ to yield TBDMSprotected dibromide 7 . The change of the $N$-protecting group was necessary because $N$-Boc protected pyrroles can undergo decomposition in the presence of strong bases used in the subsequent, two-step elimination procedure. ${ }^{20}$ Elimination of the first equivalent of hydrogen bromide from 7 was achieved by reacting 7 with 10 equivalents of $\mathrm{KO} t \mathrm{Bu}$, added as a $1 \mathrm{M}$ solution in THF at $-10^{\circ} \mathrm{C}$. This procedure not only led to the
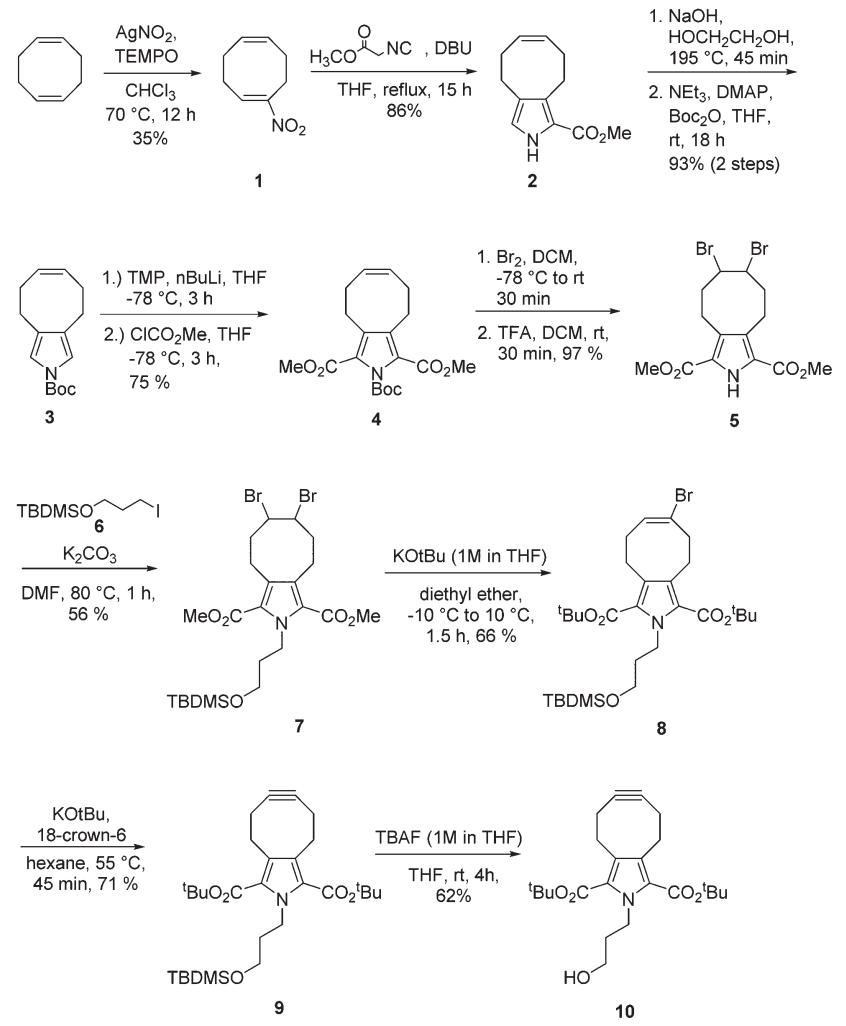

Scheme 1 Synthesis of PYRROC (10).

elimination of hydrogen bromide, but also to transesterification, providing the di-tert-butylester 8 in $66 \%$ yield. A large excess of $\mathrm{KO} t \mathrm{Bu}$ was used because test reactions with a protecting group of similar size, 2-(trimethylsilyl)ethoxymethyl (SEM), as an $N$-protecting group had shown that lesser amounts of $\mathrm{KO} t \mathrm{Bu}$ ( 1 or 5 equivalents) also did not allow the isolation of the dimethylester, but instead afforded a mixture of the methyl tert-butyl ester and monocarboxylic acids. Elimination of the second equivalent of hydrogen bromide from 8 was achieved by using $\mathrm{KO} t \mathrm{Bu}$ in the presence of catalytic amounts of 18-crown-6, providing cycloalkyne 9 in $71 \%$ yield. $^{21}$ Attempts to obtain the dimethylester derivative of 9 by using other bases such as DBU, LDA or LiOMe, or by transesterification of 9 with LiOMe, were not successful. Removal of the silyl protecting group with TBAF yielded pyrrolocyclooctyne 10 dubbed PYRROC.

In order to investigate the reaction kinetic of PYRROC (10), the second order rate constant of its reaction with benzyl azide was determined using kinetic NMR experiments. The ${ }^{1} \mathrm{H}-\mathrm{NMR}$ of the reaction between PYRROC (10) and benzyl azide shows only one peak for the benzylic protons of the resulting triazole, confirming that PYRROC indeed forms only a single compound in SPAAC with benzyl azide (ESI Fig. S1a and $\mathrm{b}_{\dagger} \dagger$ ). PYRROC displayed a second-order rate constant $k$ of $0.060 \pm$ $0.004 \mathrm{M}^{-1} \mathrm{~s}^{-1}$ in $\mathrm{CD}_{3} \mathrm{CN}$ (ESI Fig. $\mathrm{S} 1 \mathrm{c}$ and $\mathrm{d} \dagger$ ). This value is between the rate constant of $\operatorname{DIBO}^{9}\left(k=0.057 \mathrm{M}^{-1} \mathrm{~s}^{-1}\right.$ in $\mathrm{CD}_{3} \mathrm{OD}$ ), and $\mathrm{DIFO}^{8}\left(k=0.076 \mathrm{M}^{-1} \mathrm{~s}^{-1}\right.$ in $\mathrm{CD}_{3} \mathrm{CN}$ ) (Fig. 1a), both of which have proven their utility in a large number of 
biological studies. Thus, PYRROC reacts approximately six times faster than the 3,4-benzannulated cyclooctyne MOBO $\left(k=0.0095 \mathrm{M}^{-1} \mathrm{~s}^{-1}\right.$ in $\left.\mathrm{CD}_{3} \mathrm{CN}\right),{ }^{22}$ but four times slower than the 5,6-benzannulated cyclooctyne COMBO $\left(k=0.24 \mathrm{M}^{-1} \mathrm{~s}^{-1}\right.$ in $\left.\mathrm{CD}_{3} \mathrm{CN}\right){ }^{20}$ The lower reactivity of PYRROC compared to $\mathrm{COMBO}$ confirms theoretical predictions that fusion of a 5 -membered ring to the 5,6-position of cyclooctyne is less beneficial for reactivity than fusion of a 6-membered ring. ${ }^{23}$

We envision the application of symmetrically functionalized cycloalkynes such as PYRROC in the bioorthogonal, isomerfree generation of organic molecules in the cellular milieu. To test the suitability of PYRROC for this type of application in a manner as general as possible, we coupled $\mathbf{1 0}$ to the fluorophore BODIPY-FL ${ }^{24,25}$ by activating the primary hydroxyl group as a $p$-nitrophenylcarbonate, and reacting $\mathbf{1 1}$ with the primary amine 12 (Scheme 2). In the course of this study, fluorophorelabeled PYRROC 13 was subjected to SPAAC with three fluorophore azides with substantially different chemical structures: 5-TAMRA azide (14), BODIPY-TMR azide (15), and 3-azido7-hydroxycoumarin (16) (Scheme 2). The reactions were carried out in phosphate-buffered saline (PBS) to mimic the salt concentrations in a cell. The kinetics of SPAAC were analyzed via FRET (fluorescence resonance energy transfer). To allow for quantitative analysis of the reaction kinetics, each reaction was

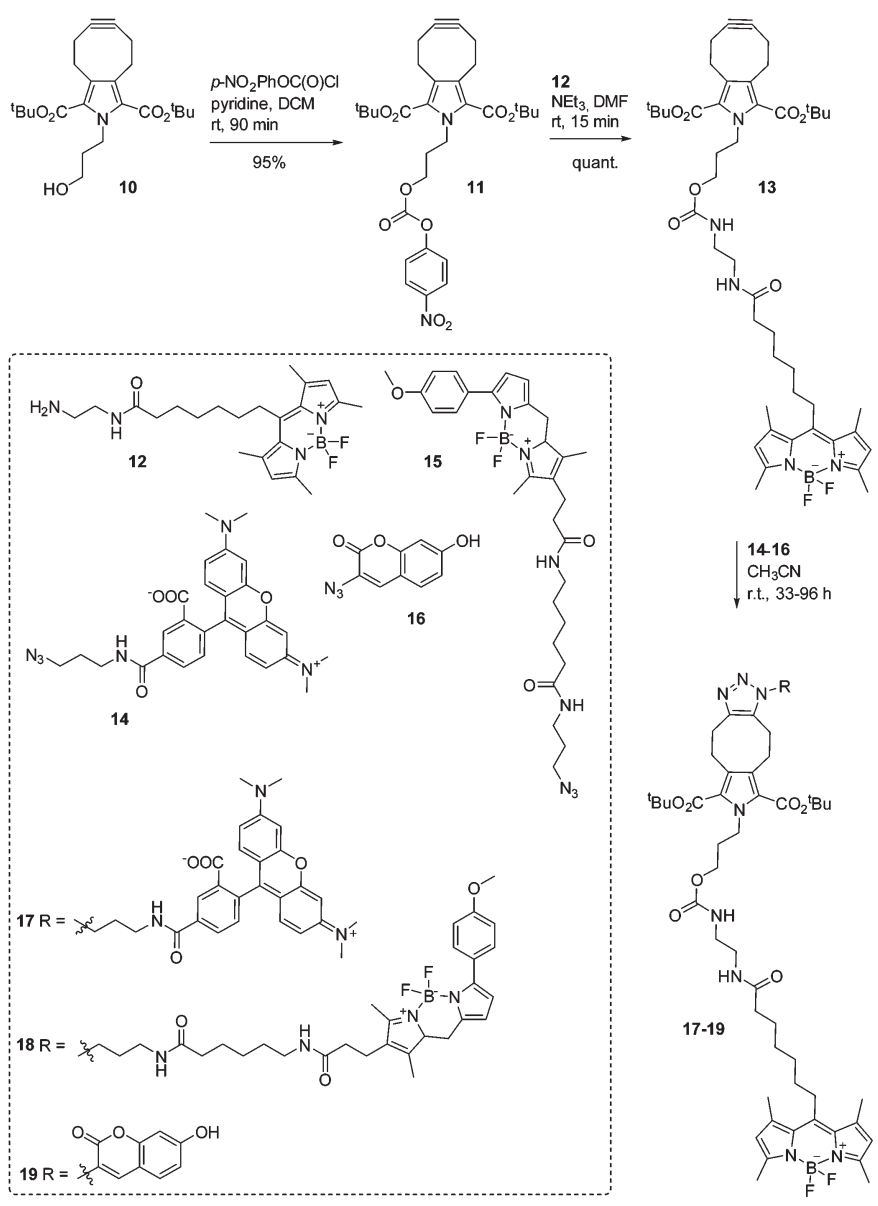

Scheme 2 Synthesis of fluorophophore-labeled triazoles 17-19. carried out in parallel with a standard curve mimicking the composition of the reaction mixture at various degrees of conversion of the reactants to the triazole products. This required prior preparation and isolation of the triazoles 17-19 on a preparative scale to allow for characterization of their fluorescence properties.

The reaction between PYRROC-BODIPY-FL (13) and 5-TAMRA azide (14) resulting in triazole 17 was found to proceed with a second-order rate constant of $13.9 \pm 0.3 \mathrm{M}^{-1}$ $\mathrm{s}^{-1}$ (ESI Fig. S2a-d $\dagger$ ), which is more than 200-fold faster than the rate constant for the reaction between PYRROC (10) and benzyl azide in acetonitrile. Using $10 \mu \mathrm{M}$ of both PYRROCBODIPY-FL (13) and TAMRA azide (14) in PBS, $74 \pm 8 \%$ of 13 was converted to the triazole 17 after $6 \mathrm{~h}$ (ESI Fig. S2e $\dagger$ ). At $50 \mu \mathrm{M}$ of both reactants, $95 \pm 1 \%$ conversion of 13 was achieved after only $2 \mathrm{~h}$.

The reaction between BODIPY-FL-labeled PYRROC (13) and BODIPY-TMR azide (15) in PBS was monitored by the decrease in intensity of the BODIPY-FL emission and the concomitant increase of intensity of the BODIPY-TMR emission by FRET (Fig. 3a and b). Using the reactants at a concentration of only $3 \mu \mathrm{M}$ in $\mathrm{PBS}$, the reaction proceeded with a second-order rate constant of $234 \pm 2 \mathrm{M}^{-1} \mathrm{~s}^{-1}$ (Fig. 3c and ESI Fig. S3c $\dagger$ ). $88 \%$ of triazole 18 was formed after $4 \mathrm{~h}$, and more than $90 \%$ after $24 \mathrm{~h}$ (Fig. 3d). This indicates that the approach is well suited to generating organic molecules in high yields at low micromolar concentrations in the aqueous environment.

In order to analyze whether PYRROC is suitable for SPAAC in the cellular milieu, we carried out the reaction in $\mathrm{K} 562$ lysates. At a concentration of $3 \mu \mathrm{M}$ of BODIPY-FL-PYRROC (13) and $3 \mu \mathrm{M}$ BODIPY-TMR azide (15), the second-order rate constant in cell lysates was determined as $17 \pm 0.3 \mathrm{M}^{-1} \mathrm{~s}^{-1}$, which is slightly more than one order of magnitude slower than the reaction in PBS (Fig. $3 c$ and ESI Fig. S3†). The slower reaction in cell lysates as compared to PBS could be due to an effective reduction of the concentration of $\mathbf{1 3}$ by non-specific reactivity with thiols, or non-covalent interactions with cell components. A more trivial potential reason is the higher viscosity of the cell lysis buffer, which, unlike PBS, contains 10\% of glycerol, and thus reduces the rate of reaction. To investigate the effect of the cell lysis buffer on the reaction rate, the reaction was carried out in cell lysis buffer without cell components. The rate constant of $16 \pm 0.3 \mathrm{M}^{-1} \mathrm{~s}^{-1}$ indicate that the reduced rate in cell lysates is caused by the components of the buffer, and not by unspecific reaction with thiols. Despite the reduced rates, more than $70 \%$ conversion was achieved in cell lysates after $24 \mathrm{~h}$ (Fig. 3d). These data place PYRROC into a promising position to mediate bioorthogonal reactions in the cellular milieu.

Reaction of BODIPY-FL-PYRROC (13) with 3-azido-7-hydroxycoumarin (16) (both at $1 \mu \mathrm{M}$ in PBS) was characterized by a second-order rate constant of $903 \pm 23 \mathrm{M}^{-1} \mathrm{~s}^{-1}$ (ESI Fig. S4 $\dagger$ ). This represents the highest rate constant of a SPAAC reaction reported to date, and is approximately 20-fold higher than the previously highest rate constant of $45.1 \mathrm{M}^{-1} \mathrm{~s}^{-1} \cdot{ }^{12}$ To analyze the influence of the BODIPY-FL substituent of PYRROC on the 

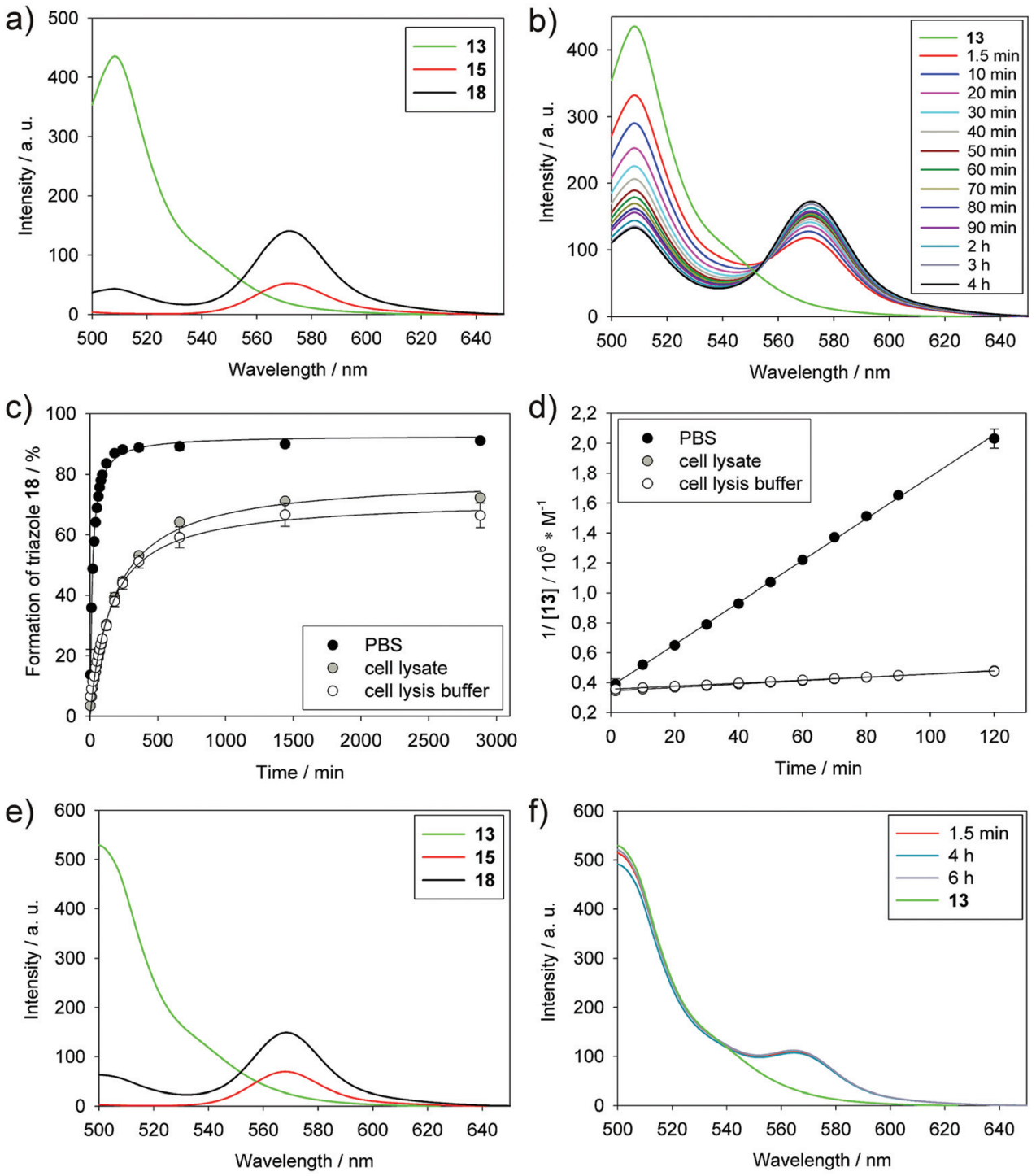

Fig. 3 Kinetics of the reaction between BODIPY-FL-labeled PYRROC (13) and BODIPY-TMR azide (15) followed by FRET. (a) Fluorescence spectra of 13, 15, and the triazole product $18(3 \mu \mathrm{M}$ each) in PBS. Excitation wavelength: $485 \mathrm{~nm}$. a. u.: arbitrary units. (b) Time course of the reaction after addition of 15 to 13 (initial final concentrations of 13 and 15: $3 \mu \mathrm{M}$ each). (c) Determination of the second-order rate constant of 13 in reaction with 15 in PBS, cell lysis buffer, and cell lysates (initial concentrations of 13 and 15: $3 \mu \mathrm{M}$ each). (d) Determination of the conversion of 13 and 15 to 18 in PBS, cell lysis buffer, and cell lysates (initial concentrations of 13 and 15: $3 \mu \mathrm{M})$. (e) Fluorescence spectra of 13, 15, and 18 (3 $\mu \mathrm{M}$ each) in acetonitrile. Excitation wavelength: $485 \mathrm{~nm}$. (f) Time course of the reaction after addition of 15 to 13 (initial concentrations of 13 and 15: $3 \mu \mathrm{M}$ each).

rate constants, we took advantage of the fact that the quantum yield of 16 increases strongly after reacting with alkynes to form triazoles. Thus, the rate of reaction between unsubstituted PYRROC 10 and 16 can also be followed by fluorescence techniques, and can thus be compared to the rate of reaction between 13 and 16. Under otherwise identical assay conditions, the reaction of 16 with unlabeled PYRROC (10) proceeded with a less than twofold lower rate constant $(k=492 \pm$ $43 \mathrm{M}^{-1} \mathrm{~s}^{-1}$, ESI Fig. $\left.\mathrm{S} 5 \dagger\right)$. These data indicate that while BODIPY-FL does contribute to the high rate constants of 13 in PBS to some extent, the majority of the high reaction rates observed in the kinetic experiments is inherent to PYRROC.
The rate constants observed with PYRROC in SPAAC in aqueous solutions are three to four orders of magnitude higher than the rate constants measured in the standard reaction of PYRROC with benzyl azide in acetonitrile. Similar rate accelerations have been observed for Diels-Alder reactions in water compared to nonpolar solvents. ${ }^{26,27}$ They are typically rationalized by a hydrophobic effect, leading to pre-association of the organic molecules in order to minimize their exposure to water, and reduction of the hydrophobic surface area in the course of the reaction. ${ }^{28}$ The difference in rate constants in the reactions of 13 with the aliphatic azides 14 and 15 can be rationalized by the increased hydrophobicity of $\mathbf{1 5}$. However, the higher rate constants obtained with $\mathbf{1 6}$ compared to $\mathbf{1 4}$ 
and 15 cannot be explained with its hydrophobicity; instead, it might reflect a higher intrinsic reactivity of $\mathbf{1 6}$ caused by the conjugation of the azide moiety with the phenolic system, which is partially deprotonated at the neutral pH of PBS.

Rate enhancements in SPAAC reactions as a result of shifting to a more polar solvent or solvent mixture have also previously been reported, ${ }^{10,12,29}$ but the effect has not been so pronounced. This is presumably because the SPAAC reaction rate determinations have been carried out in partly aqueous solutions only.

In order to validate the aqueous buffer as the underlying cause of the high reaction rates, we carried out the reaction between 13 and 15 in acetonitrile and followed it by analysis of the fluorescence spectra. Using the same concentrations $(3 \mu \mathrm{M})$ of cycloalkyne 13 and azide 15, no significant reaction was observed after $6 \mathrm{~h}$ (Fig. 3e and $\mathrm{f}$ ). In contrast, the reaction in PBS had shown significant progress after just 90 seconds, and was virtually complete after only $4 \mathrm{~h}$ (Fig. $3 \mathrm{~b}$ ). This demonstrates that the aqueous buffer is a major contributor for the high reaction constants in SPAAC.

In conclusion, we have presented PYRROC as the first functionalized cycloalkyne that cannot form isomers in SPAAC. The design concept of PYRROC facilitates a new field of application for SPAAC: the creation of uniform organic molecules, with a molecular weight that is prohibitively high for cell permeability, in the cellular environment. PYRROC displays unprecedented reaction rates in the reaction with azides in aqueous systems, and is suitable for derivatization at the hydroxy group. The absence of charged chemical moieties in PYRROC is likely to contribute to good cell permeability of its conjugates with organic molecules. No limitations with respect to the selection of azides have been observed to this point. We believe that the concept of symmetrically substituted cycloalkynes, as exemplified by PYRROC, will strongly influence future research in the fields of bioorthogonal chemistry, chemical biology, and medicinal chemistry. Applications of PYRROC in cell-based assays will be reported in due course.

This work was supported by the Deutsche Forschungsgemeinschaft (BE4572/2-1). We extend our thanks to Lothar Hennig for support with the NMR-based kinetic analysis, Angela Berg for experimental support, and Ralf Hoffmann and Thole Züchner for providing access to their fluorescence spectrometer.

\section{Notes and references}

1 R. Huisgen, Angew. Chem., Int. Ed. Engl., 1963, 2, 565-598.

2 V. V. Rostovtsev, L. G. Green, V. V. Fokin and K. B. Sharpless, Angew. Chem., Int. Ed., 2002, 41, 25962599.

3 C. W. Tornoe, C. Christensen and M. Meldal, J. Org. Chem., 2002, 67, 3057-3064.

4 M. Meldal and C. W. Tornoe, Chem. Rev., 2008, 108, 29523015.
5 G. Wittig and A. Krebs, Chem. Ber., 1961, 94, 3260-3275.

6 J. C. Jewett and C. R. Bertozzi, Chem. Soc. Rev., 2010, 39, 1272-1279.

7 N. J. Agard, J. A. Prescher and C. R. Bertozzi, J. Am. Chem. Soc., 2004, 126, 15046-15047.

8 J. M. Baskin, J. A. Prescher, S. T. Laughlin, N. J. Agard, P. V. Chang, I. A. Miller, A. Lo, J. A. Codelli and C. R. Bertozzi, Proc. Natl. Acad. Sci. U. S. A., 2007, 104, 16793-16797.

9 N. E. Mbua, J. Guo, M. A. Wolfert, R. Steet and G. J. Boons, ChemBioChem, 2011, 12, 1912-1921.

10 J. Dommerholt, S. Schmidt, R. Temming, L. J. Hendriks, F. P. Rutjes, J. C. van Hest, D. J. Lefeber, P. Friedl and F. L. van Delft, Angew. Chem., Int. Ed., 2010, 49, 94229425.

11 F. Starke, M. Walther and H. J. Pietzsch, ARKIVOC, 2010, 350-359.

12 C. D. McNitt and V. V. Popik, Org. Biomol. Chem., 2012, 10, 8200-8202.

13 M. F. Debets, S. S. van Berkel, J. Dommerholt, A. T. Dirks, F. P. Rutjes and F. L. van Delft, Acc. Chem. Res., 2011, 44, 805-815.

14 A. Rutkowska and C. Schultz, Angew. Chem., Int. Ed., 2012, 51, 8166-8176.

15 J. A. Wells and C. L. McClendon, Nature, 2007, 450, 10011009.

16 S. Maity, S. Manna, S. Rana, T. Naveen, A. Mallick and D. Maiti, J. Am. Chem. Soc., 2013, 135, 33553358.

17 D. H. R. Barton and S. Z. Zard, J. Chem. Soc., Chem. Commun., 1985, 1098-1100.

18 A. Fürstner, H. Krause and O. R. Thiel, Tetrahedron, 2002, 58, 6373-6380.

19 T. J. Donohoe, C. E. Headley, R. P. Cousins and A. Cowley, Org. Lett., 2003, 5, 999-1002.

20 B. R. Varga, M. Kallay, K. Hegyi, S. Beni and P. Kele, Chemistry, 2012, 18, 822-828.

21 C. Antony-Mayer and H. Meier, Chem. Ber., 1988, 121, 2013-2018.

22 E. M. Sletten, H. Nakamura, J. C. Jewett and C. R. Bertozzi, J. Am. Chem. Soc., 2010, 132, 11799-11805.

23 K. Chenoweth, D. Chenoweth and W. A. Goddard 3rd, Org. Biomol. Chem., 2009, 7, 5255-5258.

24 I. A. Boldyrev and J. G. Molotkovsky, Russ. J. Bioorg. Chem., 2006, 32, 78-83.

25 N. Elumalai, A. Berg, K. Natarajan, A. Scharow and T. Berg, Angew. Chem., Int. Ed., 2015, DOI: 10.1002/anie.201410672.

26 S. Otto, W. Blokzijl and J. B. F. N. Engberts, J. Org. Chem., 1994, 59, 5372-5376.

27 D. C. Rideout and R. Breslow, J. Am. Chem. Soc., 1980, 102, 7816-7817.

28 S. Otto and J. B. Engberts, Org. Biomol. Chem., 2003, 1, 2809-2820.

29 X. Ning, J. Guo, M. A. Wolfert and G. J. Boons, Angew. Chem., Int. Ed., 2008, 47, 2253-2255. 